\title{
Metodología de Elkonin-Davidov y la teoría de la actividad en América Latina
}

\author{
Elkonin-Davidov Metodology and activity theory in Latina America
}

\author{
Yulia Solovieva ${ }^{1}$ \\ Luis Quintanar ${ }^{2}$
}

\begin{abstract}
RESUMEN
La teoría de la actividad continua la aproximación histórico-cultural, introducida por L. S. Vigotsky. Dentro de la teoría de la actividad, se puede hablar de varias concepciones más paprticulares que, a veces, se tienden a consebir de forma aislada. La concepción de Elkonin-Davidov puede servir como un ejemplo. El objetivo del artículo es mostrar que la teoría de la actividad psicológica ofrece una visión dialéctica del proceso de la enseñanza y aprendizaje. En lugar de las aplicaciones aislantes de una de las concepciones estrechas, el artículo propone la integración de las aportaciones de la teoría de la actividad como una base experimental para la organización de la enseñanza que conduce al desarrollo psychológico en América Latina.
\end{abstract}

Palabras clave: Sistema de ElkoninDavidov. Enseñanza y desarrollo. Teoría de la actividad. Metodología de enseñanza. Teorías de educación.

\begin{abstract}
Activity theory continues historicalcultural approach introduced by L.S. Vigotsky. Within activity theory, it's possible to peek about particular conceptions, which, sometimes, might be understood in isolate way. Educational system of Elkonin-Davidov might serve as an example of such conceptions. The goal of the article is to show that activity theory offers a dialectic vision of the process of teaching and learning. Instead of isolated usage of one of stretch conceptions, the article proposes an integration of contributions of activity theory as an experimental base for organization of teaching process, which leads to psychological development in Latin America.
\end{abstract}

Keywords: System Elkonin-Davidov. Teaching and development. Activity theory. Methods of teaching. Educational theories.

\footnotetext{
1 Universidad Autónoma de Tlaxcala, Universidad Autónoma de Puebla, Orcid: https://orcid.org/0000-000156101474. Email: aveivolosailuy@gmail.com.

2 Universidad Autónoma de Tlaxcala, Universidad Autónoma de Puebla, Orcid: https://orcid.org/0000-0002-9758-1467. Email: ranatniuq@icloud.com.
} 
“...una auténtica solución de los problemas de la educación ... presupone un cambio del tipo de pensamiento... mediante el contenido de las disciplinas y los métodos de su enseñanza.

V.V. Davidov

\section{Introducción}

El enfoque histórico-cultural, introducido en la psicología por L.S. Vigotsky, considera que el proceso de la adquisición de la experiencia históricocultural es la fuente principal del desarrollo del niño (VIGOTSKY, 1996; ELKONIN, 1989; OBUKHOVA, 2006).

En la concepción de Vigotsky, se establece una relación particular entre los procesos de enseñanza y desarrollo. Vigotsky ha determinado que existe una estrecha relación entre los procesos de la enseñanza y aprendizaje, pero que estos procesos no coinciden (VIGOTSKY, 1991). Este autor señala que la enseñanza determina el desarrollo y conduce hacia él a través de interacción en la zona del desarrollo próximo. Lo anterior implica que el desarrollo del niño realmente depende de la forma de la enseñanza y del método eligido por los maestros. Podemos decirlo con otras palabras aplicadas al tema de enseñanza-aprendizaje: "así como es la enseñanza, será el desarrollo del niño”. No toda la enseñanza necesariamente es positiva y no siempre conduce al desarrollo, lo cual implica la necesidad de investigar y organizar el proceso de la enseñanza. Acerca de la importancia del proceso de enseñanza-aprendizaje para el desarrollo psicológico, Vigotsky (1991) escribió que la enseñanza puede interferir en el transcurso del desarrollo de manera decisiva, debido a que las funciones, en el momento inicial del proceso enseñanza-aprendizaje, aún no han madurado, mientras que la enseñanza puede organizar el proceso de su desarrollo y, de este modo, determinar su destino.

La concepción de la enseñanza que conduce al desarrollo, propuesta por Vigotsky (1991, 1992), se ha precisado posteriormente a través del diseño metodológico aplicado a la realidad del desarrollo y la enseñanza. En particular, esto se relaciona con los trabajos de Galperin (2000) y Davidov (2000) y sus colaboradores. En estos trabajos se demuestra que el papel del 
investigador, entonces, no es solo observar y registrar, sino garantizar de manera óptima este proceso de adquisición, en el cual el lugar principal lo ocupa el niño mismo, junto con el educador y el maestro.

Ambas posturas se basan en la teoría de la actividad que continúa con la investigación y aplicación de esta idea fundamental de L.S. Vigotsky acerca de la relación entre la enseñanza y el desarrollo.

De acuerdo con la teoría de la actividad, el proceso de enseñanza-aprendizaje se concidera como un proceso dinámico y dialéctico que incluye a ambos participantes o agentes sociales: maestros y alumnos. Consideramos que, actualmente, una innovación educativa es necesaria y emergente que tenga en cuenta la participación integral de estos integrantes desde la modificación integral de toda su metodología (QUINTANAR y SOLOVIEVA, 2020).

La teoría de la actividad incluye la consideración de los elementos estructurales de cada actividad, tales como el objeto y el objetivo de la actividad, el motivo, las operaciones y la base orientadora (TALIZINA, 2018, 2019). Al mismo tiempo, la teoría de la actividad permite considerar todo el proceso educativo como un sistema, en el cual activamente participan varios agentes sociales: alumnos y maestros (SOLOVIEVA, 2019).

Dentro de la teoría de la actividad han surgido distintas concepciones particulares que han tenido importancia para algunas áreas de aplicación de conocimiento. Una de estas concepciones de la concepción de la formación de la acción mental por etapas propuesta por Galperin (2000) y la que fue aplicada inicialmente en el campo de la enseñanza en la escuela primaria regular como una concepción general (TALIZINA, 1984) y cómo base de trabajo con las disciplinas o materias escolares particulares de las matemáticas, procesos físicos y geometría (TALIZINA, SOLOVIEVA y QUINTANAR, 2017; NICOLA y TALIZINA, 2017). Existen trabajos de investigación que fueron dirigidos a la enseñanza del idioma extranjero (SOLOVIEVA, 2015), a la imaginación y desarrollo intelectual del niño en la edad preescolar (OBUKHOVA, 2006).

Fue Talizina quien ha logrado consolidar y unir estas diferentes aportaciones en una sólida versión teórico-metodológica de la "teoría de la actividad aplicada a la 
enseñanza", como se denomina su libro publicado únicamente en el idioma español (TALIZINA, 2019) y su último libro en el idioma ruso (TALIZINA, 2018). En estos trabajos se presenta una versión sistematizada de la posibilidad de considerar al proceso de enseñanza-aprendizaje como una actividad psicológica, es decir, como actividad rectora de la enseñanza-aprendizaje dirigido en la edad escolar.

La concepción psicológica sobre la edad escolar, que en particular nos interesa en este escrito, es la concepción de la enseñanza que conduce al desarrollo (traducida al idioma español y portugues como la "enseñanza desorralloradora"), expuesta de forma teórica y metodológica en los trabajos de Davidov (1998, 2000). A esta concepción se le conoce como "sistema de Davidov-Elkonin" en las publicaciones en ruso hasta el último año (VORONTSOV, 2019) o, de acuerdo con las últimas publicaciones en el idioma portugues, como el "sistema Davidov-Elkonin-Repkin" (PUENTES, 2018; PUENTES y LONGAREZI, 2020). En particular, se trata de una obra monumental, un libro de Davidov, el cual debería ser traducido como "Enseñanza-aprendizaje que conduce al desarrollo" (DAVIDOV, 2000). En forma abreviada, podría ser traducido como "enseñanza que conduse al desarrollo".

Queremos mencionar, de paso, que el tema de traducción de la palabra rusa "obucheniye" al idioma español y portuguéz, sigue siendo un tema de discusión (PUENTES y LONGAREZI, 2020). De acuerdo a nuestra opinión, la más oportuna traducción de este término es "enseñanza", debido a que la palabra "ucheniye" quiere decir "aprendizaje" en ruso. El término "obucheniye" (nombre de la revista, en la cual estamos publicando nuestro artículo con gran entusiasmo), el algunos ocntextos puede significar "enseñanza-aprendizaje”, pero nunca (en ningún contexto) puede significar "aprendizaje"2.

El objetivo de este artículo es consideraremos los elementos esenciales de esta concepción en relación con las posiciones principales de la teoría de la actividad en general.

\footnotetext{
2 El primer autor de este artículo es de origen ruso con Master en Ciencias Históricas y Doctorado en Psicología, alumna directa y colaboradora de N.F Talizina. Esperemos que esta referencia dará cierta validez a la traducción de los términos, utilizados en los textos de los representantes de la teoría de la actividad.
} 


\section{Concepción de enseñanza-aprendizaje que conduce al desarrollo}

De acuerdo con Davidov, la actividad rectora de la edad escolar es la actividad del aprendizaje escolar dirigido, la cual, en ocasiones también se traduce como "actividad de estudios". Esta actividad rectora en el idioma ruso suena como "deyatelnost ucheniya" ili "uchebnaya deyatelnost". Al mismo tiempo, existe la pabara "obucheniye". Queremos señalar que en el idioma ruso se utiliza una única palabra "obucheniye", a propósito, es el título de la revista en la que se publica este artículo. Esta palabra, en ciertos contextos, incluye simultáneamente los términos tanto la enseñanza como el aprendizaje, por lo cual pueden surgir algunas complicaciones a la hora de traducir el término a otros idiomas. El traductor siempre tiene que elegir entre traducir este término solo como "enseñanza" o como proceso de "enseñanzaaprendizaje". "Obucheniye”, puede implicar ambos procesos de la enseñanza y del aprendizaje, pero más específicametne se enfoca en el aspecto de la enseñanza, es decir, en la actividad del maestro, quien les enseña a los alumnos.

Entonces, la actividad rectora del niño, en la edad escolar, es el aprendizaje dirigido, mientras que la actividad profesional del adulto es la enseñanza que conduce al desarrollo ("razvivayuschee obucheniye"). Cabe señalar que estas actividades pueden ser consideradas por separado con fines de estudios, sin embargo, en realidad son interdependientes y una no puede existir sin la otra. La actividad de aprendizaje escolar del alumno no se puede llevar a cabo sin la actividad de la enseñanza que conduce al desarrollo de este mismo alumno.

Sin duda alguna, Davidov (2000), al proponer este término que se asocia actualmente con toda con su concepción (VORONTSOV, 2019), de la enseñanza desarrolladora o de la enseñanza que conduce al desarrollo pensaba en la concepción de Vigotsky acerca de la zona del desarrollo próximo (VIGOTSKY, 1991). Es decir, se trata de una concepción de la enseñanza que constantemente conforma la zona del desarrollo próximo de los alumnos o que transcurre en la zona del desarrollo próximo. Es aquella enseñanza que siempre introduce algo nuevo, se mueve hacía adelante y no se estanca en las metas ya logradas.

Davidov, en sus escritos, analiza con detalles el contenido de la actividad de la enseñanza y del aprendizaje como parte íntegra del proceso educativo. Este 
autor establece el objetivo fundamental de la edad escolar que consiste en introducción y gradual formación de la visión teórica del mundo. La vía de esta formación es teórico-reflexiva y su contenido de esta formación es el trabajo con los conceptos centíficos (teóricos) de áreas de conocimientos.

En este sentido, es necesario recordar que Davidov (2000) cita a Marx y Engels en muchas ocasiones, subrayando que las ciencias surgieron como una necesidad de generalización, abstracción y sistematización de conocimiento. Vigotsky (1992) también cita a Marx, cuando habla de la necesidad de formación de conceptos científicos, diciendo que, en las ciencias, la apariencia no coincide con la esencia de las cosas y fenómenos de la naturaleza y la sociedad.

Para precisar esta idea, Davidov propone aclarar que es necesario diferenciar el término "aprendizaje" como un término general, del término de la "actividad de aprendizaje escolar" como un término psicológico particular. El "aprendizaje" puede acompañar y surgir durante la realización de diversas actividades, tales como comunicación, deporte, tareas cotidianas, etc. Este aprendizaje no necesariamente es teórico ni es reflexivo y no se conforma de una forma sistemática ni organizada.

En el caso de la actividad rectora del aprendizaje escolar, el proceso de aprendizaje es determinadamente organizado, es un proceso que puede ser planeado y estudiado con detalles. El objetivo de este aprendizaje es adquirir las bases de pensamiento teórico-reflexivo por parte de cada alumno participante. Este tipo de pensamiento se diferencia de pensamiento empírico, porque transcurre sobre la base de sistemas adquiridos de conceptos. Este pensamiento es el resultado del aprendizaje y no puede existir sin una actividad adecuada del maestro, sin la enseñanza que conduce al desarrollo.

Para Davidov (1998), aquella enseñanza que conduce al desarrollo es la que puede ser organizada sobre la base de estas premisas dialécticas y materialistas. Este autor, con sus seguidores, propone organizar, sistematizar, estudiar y perfeccionar constantemente esta actividad en forma de una contínua investigación psicológica educativa (SIDNEVA, 2020). "Conducir al desarrollo" o ser una enseñanza desarrolladora" se debe comprender como aquella enseñanza que logra introducir y sistematizar los conocimientos teóricos de los alumnos. 
Como objetivo del proceso educativo en la escuela, de acuerdo a la aproximación de L.S. Vygotsky y sus seguidores, se plantea la introducción y la adquisición de conceptos científicos de las ciencias básicas: lingüística, matemáticas, ciencias sociales y naturales (DAVIDOV, 2000). Para cumplir con este objetivo, es necesario diferenciar entre conceptos cotidianos (empíricos) y científicos (teóricos). Antes que nada, dicha diferenciación debe ser clara para los maestros, para que ellos puedan lograr una formación conceptual clara en sus alumnos.

A diferencias de los conceptos cotidianos, los conceptos teóricos siempre formas sistemas conceptuales complejos. El niño no puede adquirir los conceptos teóricos por memorización o de forma aislada y desconectada, sino que debe adquirir todo un sistema conceptual jerarquizado. Es necesario comprender que los conceptos no se adquieren a través de la simple interacción con los objetos. Si esto fuera así, la humanidad no hubiera necesitado de la ciencia y se hubiera satisfecha solo con la práctica cotidiana.

Desde la teoría de la actividad, apropiadamente comprendida, estas ideas no simplemente son erróneas e inconsistentes, sino peligrosas para la práctica educativa. Así, desde la teoría de la actividad que sigue las ideas expuestas por Vygotsky, se plantea que se debe establecer una "ruptura" entre los conceptos cotidianos y científicos (VIGOTSKY, 1995). Esta ruptura es absolutamente necesaria, porque los conceptos "científicos no crecen desde los conceptos cotidianos", sino que se introducen especialmente a partir de las acciones especiales y dirigidas por el maestro. Repetimos de nuevo: los conceptos cotidianos no se transforman en los conceptos científicos, porque se trata de otro tipo de conceptos. El niño no construye los conceptos científicos, sino solo puede adquirirlos en las acciones intelectuales especialmente organizadas e introducidos por el adulto.

Podemos decir que, para la formación apropiada de los conceptos teóricos, el adulto tiene que presentar claramente los componentes de las características esenciales del concepto con el que trabaja en el momento dado de su proceso educativo. De esta forma, es necesario que los maestros comprendan y les transmitan a los alumnos que las características esenciales de los conceptos científicas son el resultado de procesos de abstracción y generalización de las 
diversas características que tienen los objetos y fenómenos del mundo, pero que no todas estas características forman parte del concepto científico.

Debido a lo anterior, la formación de los conceptos teóricos requiere de esfuerzo intelectual particular, en el cual el maestro debe servir de una guía y orientación para los alumnos. Si nosotros deseamos optar por una vía menos dolorosa y frustrante para los alumnos, debemos reconsiderar toda la metodología de la enseñanza y comprender que esta requiere de una organización especial. Además, podemos expresar que la organización especial de la metodología de la enseñanza se debe considerar como un proceso creativo, contínuo y flexible y que no puede existir un final de este proceso, tal y como no pude existir un final en la creación de una tecnología y arte en la humanidad. La experimentación y la innovación en la metodología de la enseñanza debe ser tratada como algo permanente y dinámico. Esta experimentación y la innovación se puede realizar solamente bajo una adecuada comprensión del concepto de la orientación como elemento control de la actividad de enseñanza-aprendizaje que se retoma del sistema psicológico de P.Ya. Galperin (SOLOVIEVA Y QUINTANAR, 2018a, 2019).

De acuerdo con Davidov, el objetivo de la enseñanza escolar es la introducción de los conceptos teóricos generales de las materias básicas que se enseñan en la escuela primaria, sin importar con qué nombre sean llamandas en cada escuela o en cada región: materias exactas, materias linguísticas, materias naturales y materias sociales. El conocimiento científico o teórico forma parte de un bagaje cultural complejo que se transmite a través de los medios culturales ideales (ILIENKOV, 2009) y conforma un plano ideal de la cultura.

La formación de los conceptos teóricos no se puede dar en una forma espontánea, sino requiere de esfuerzo intelectual particular, en el cual el maestro debe servir de una guía y orientación para los alumnos. El esfuerzo implica una elección y diseño de las acciones intelectuales, en las cuales los conceptos que se adquieren se van a utilizar. Dicha idea se basa en la argumentación de Davidov (1996). De acuerdo con este autor, no puede existir una separación entre el concepto y la acción. Cada concepto científico no se utiliza en cualquier tipo de acción, sino en una acción intelectual. El éxito de la 
enseñanza depende, entre otras cosas, de una adecuada elección de las acciones intelectuales para cada concepto que se forma en un nivel educativo particular.

Para lograr con los objetivos tan elevados en la enseñanza escolar, se propone una transformación completa de las materias escolares junto con los métodos que se utilizan en la primaria, es decir, se debe realizar la transformación de toda la didáctica escolar. Lo anterior, obviamente, implica un trabajo previo teórico y práctico de una preparación especial de los maestros. Esta tarea fue implementada en la escuela pública N. 91 de la ciudad de Moscú, N. 17 de Jarkov y N.11 de Tula en la URSS, iniciada bajo la dirección personal de V.V. Davidov y D.B. Elkonin, quienes organizaron e impulsaron este trabajo con el grupo de colaboradores (DAVIDOV, 2000). El trabajo fue continuado por sus seguidores y alumnos posteriormente y se lleva a cabo hasta el día de hoy (VORONTSOV, 2019).

El programa de trabajo de la escuela de Elkonin-Davidov se conoce actualmente como una metodología particular y original y cómo una de las propuestas que realiza los planteamientos de Vygotsky sobre la relación estrecha que existe entre el proceso de la enseñanza y el desarrollo (VIGOTSKY, 1992). Es muy interesante que, en el último programa publicado de este modelo didáctico, claramente se escribe que se trata de un programa realizado desde el enfoque de la teoría de la actividad:

El hecho de garantizar las condiciones para la conformación del niño como la del sujeto de la actividad de aprendizaje escolar (actividad de estudio $^{3}$ ), interesado en un cambio de sí mismo y capaz para realizar este cambio, - este es el objetivo principal de cada materia escolar, lo cual, indudablemente, es la realización de la aproximación de la teorá de la actividad (VORONTSOV, 2019, p. 33).

Los rasgos principales de este sistema de enseñanza, consisten en la formación del conocimiento sistémico, teórico, ante el cual, el material escolar no se va a memorizar, sino adquirirse en el proceso de su comprensión gradual. En todo momento, los escolares deben darse cuenta de sus propias acciones, deben conscientizarlas, deben realizar no sólo las operaciones ejecutivas y reproductoras, sino deben tener la reflexión de toda su actividad. En esta parte, es lamentable que

\footnotetext{
${ }^{3}$ En el idioma portugués se conoce más el término "actividad de estudio" que, nos parece, es mejor traducir como "actividad de aprendizaje escolar". Nota de autores.
} 
los autores del programa no citan a Galperin, pero, en realidad, se trata de los conceptos introducidos por este autor. Fue Galperin (2000) quien escribió, que no hay nada mejor que preguntarle al alumno, sobre qué hace y porqué lo hace, para garantizar una constante conscientización de su actividad intelectual. Se trata de una característica de la acción reflexiva y consciente.

De acuerdo a nuestra opinión, la concepción de Galperin se encuentra incluida y considerada dentro de la de la "enseñanza desarrolladora" como su eje general. El hecho de que la postura de Galperin (2000) y Talizina, (2018, 2019), aparentemente más teórica y más general, en realidad, es justo la necesaria para comprender la aproximación hacía la enseñanza-aprendizaje como una actividad de los alumnos orientada y guiada por el maestro y que permite la adquisición gradual de los sistemas de conceptos teóricos. La postura de Galperin y Talizina permite realizar consecuentemente los principios siguientes: 1) formación de la acción intelectual por etapas; 2) elaboración de la base orientadora de la acción; 3) trabajo con problemas intelectuales como problemas creativos que garantizan la formación del pensamiento teórico-reflexivo (ROSAS y SOLOVIEVA, 2018). Estos principios son únicos y no se encontraban elaborados por parte de otros seguidores de Vigotsky en el campo educativo (SOLOVIEVA, 2019).

En la concepción de Elkonin-Davidov, se le dio la prioridad al tercer principio: al trabajo con los problemas intelectuales creativos. Trataremos, a continuación, las particularidades principales de la concepción de Elkonin-Davidov.

\section{Las particularidades del sistema de Elkonin-Davidov}

Las diferencias principales entre la actual expresión de programa de ElkoninDavidov y la teoría de Galperin, de acuerdo a nuestra opinión, consisten en: ausencia de énfasis metodológico en las etapas de formación de la acción y la ausencia de aplicación práctica del concepto de la "base oreintadora de la acción". Al mismo tiempo, este programa se caracteriza por la elaboración detallada y minusiosa de las didácticas para las materias escolares del nivel primaria y secundaria (VORONTSOV, 2019). Estos programas se aplican actualmente en las escuelas que trabajan de acuerdo al programa de Elkonin-Davidov en la ciudad de Moscú. 
¿Cuáles son las consecuencias de estas diferencias para la aplicación del enfoque de la teoría de la actividad para la enseñanza en América Latina?

En primer lugar, la ausencia de la introducción explícita de la acción intelectual desde la etapa de la acción materializara puede complicar bastante la comprensión y la reflexión de los alumnos en los primeros tres grados de la escuela primaria. La ausencia de la etapa materializada se refleja en el uso de las fórmulas generalizados desde el inicio de los es la propuesta más radical del sistema de Davidov-Elkonin. En las matemáticas no se utilizan cifras, sino las determinaciones algebráicas y simbólicas entre las magnitudes. En la enseñanza del idioma se utiliza la transcripción fonética tradicional en lugar de las letras.

Tal situación es específicamente complicada para los niños que carecen de un nivel óptimo de la preparación psicológica para la escuela, lo cual es bastante común en los países de la América Latina. Se refiere al bajo nivel de adquisición de la función simbólica, desarrollo amplio del lenguaje oral y la imaginación, lo cual han mostrado nuestros múltiples estudios previos (SOLOVIEVA y QUINTANAR, 2013; GONZÁLES-MORENO y SOLOVIEVA, 2016, 2019; DE MORAES, 2019). Los niños con bajo nivel de preparación para la escuela no pueden incluirse de inmediato en la solución de problemas creativos en el plano perceptivo o verbal.

Al mismo tiempo, no se proponen las etapas formativas del plano de introducción de una acción, tal y cómo esto se hace por parte de los seguidores de P. Ya. Galperin y N.F. Talizina. En el sistema de Davidov, se inicie desde la solución de los problemas desde el plano perceptivo que nosotros llamamos como plano generalizado en una reciente publicación (SOLOVIEVA y QUINTANAR, 2020a). Se trata de un nivel perceptivo que implica trabajo con formulas y esquemas desde el inicio, sin pasar por el nivel de trabajo con los esquemas externos materializados, lo cual no se puede considerar como una situación educativa para niños que recien inicien el aprendizaje en la escuela primaria.

En segundo lugar, la ausencia de uso práctico del concepto de la "base orientadora de la acción" y su sustitución práctica por la "solución de problemas creativos" conduce a la ausencia de un método organizado y generalizado para el trabajo de los docentes, específicamente, para el nivel de la escuela primaria. Sin una 
elaboración detallada de este concepto, los maestros se quedan a la propia suerte y su trabajo puede ser demasiado caótico y desorganizado. La solución de problemas creativos con una previa orientación teórica no resulta efectiva en las condiciones de baja preparación profesional de los maestros tanto en el plano de la disciplina, como en los conocimientos teóricos de la psicología de Vigotsky y la teoría de la actividad.

La complicación consiste en la ausencia de una precisa orientación por parte del maestro para que los niños puedan realizar las acciones matemáticas o de análisis de las palabras. Si este aspecto no se descarta, más bien, no se hace énfasis en la necesidad de elaboración de una base orientadora de la acción única para todos los alumnos. Se busca, implícitamente, que los alumnos logren solucionar los problemas a través de una iniciativa o una adivinanza. Para mucho niños esta enseñanza resulta ser insoportable, porque no logran comprender qué es lo que piden los maestros. Además, el concurso para entrar a esta escuela es muy alto y es muy difícil ser admitido sin una preparación adicional de los niños.

De todo lo mencionado, lo más preocupante es aspecto más complicado es la ausencia de análisis de una planeación sistematizada para pasar de análisis de las palabras orales del idioma ruso a las palabras escritas. Las letras se introducen al final, después de realizar el análisis de los fonemas que conforman a las palabras. Las letras se agregan de una forma voluntarista al final de este proceso, y los niños cometen muchos errores en la escritura del idioma ruso. La complicación en este idioma es que, igual que en el idioma portugués, existen vocales llamados reducidos que se pronuncian de una forma distinta en dependencia de su acento tónico. Cuando se pasa a letras, obviamente los niños cometen errores, porque escuchan sonidos diferentes en dependencia de acento tónico. Un ejemplo en el idioma portugués sería la palabra "pato", en la cual se escribe "o", pero se pronuncia una vocal poco clara que se acerca a "u". Este problema se puede evitar a partir de elaboración de una base orientadora de la acción que les permite a los niños analizar el cambio del sonido verbal en dependencia del acento en la palabra. Sin embargo, la base orientadora única de la acción de análisis fonético y su realización por las etapas no se realiza. Los autores de este artículo han elaborado el método de análisis fonético inicial de las palabras, así como sistematización de la relación 
entre fonema y grafema en los idiomas español (SOLOVIEVA y QUINTANAR, 2017) e inglés (SOLOVIEVA, 2015), considerando el trabajo formativo a partir de introducción de la base orientadora de la acción intelectual de análisis reflexivo de las palabras y la formación de esta acción por etapas: materializada, perceptiva generalizada, verbal y, finalmente, escrita (SOLOVIEVA y QUINTANAR, 2020a).

Desde nuestro punto de vista, es útil analizar las fortalezas y las debilidades del sistema Davidov-Elkonin y la posibilidad de su aplicación. La modificación de esta metodología es indispensable y, desde nuestro punto de vista, debe incluir necesariamente la inclusión de la base orientadora de la acción y de las etapas de introducción de la acción intelectual, específicamente en los primeros grados de la escuela primaria.

A esta consideración debemos agregar que, en los países de América Latina, existen serías desventajas que se reflejan en el desarrollo psicológico de los niños y que deben ser consideradas durante la utilización de los métodos educativos.

En el primer lugar, es el bajo nivel de preparación de niños para los estudios escolares, debido a ausencia de la actividad rectora de juego temático de roles, actividad gráfica y literaria amplia que son fundamentales para garantizar un alto nivel de preparación del niño para la escuela (SOLOVIEVA y QUINTANAR, 2013; GONZÁLES-MORENO y SOLOVIEVA, 2019).

En el segundo lugar, es el bajo nivel de preparación profesional de los maestros de las escuelas públicas y particulares (ROSAS y SOLOVIEVA, 2019). Los maestros, con frecuencia, no dominan los sistemas de conceptos, ni tampoco pueden formular los problemas creativos que el sistema de Galperin y Elkonin-Davidov propone.

Nuestra propuesta alternativa, a la cual invitamos a unirse a todos los interesados y excelentes investigadores en Brasil y América Latina, se trata de organizar la escuela primaria predominantemente en base a las ideas de Galperin y Talizina y, posteriormente, con los mismos alumnos (siempre y cuando hayan pasado por estos niveles de la enseñanza), utilizar predominantemente la propuesta del sistema de Elkonin-Davidov para la escuela secundaria, el método que debe ser basado en la resolución de problemas creativos y las investigaciones por iniciativa de los propios 
alumnos. En el nivel de la escuela secundaria ya sería más factible pasar a la aplicación del método de solución y creación propia de los problemas intelectuales variables en los planos perceptivo y verbal de la acción. Lo anterior podría ser viable, siempre y cuando, ya existan las bases de los conceptos y orientaciones teóricas fundamentales.

Queremos mencionar algunos aspectos concretos y originales de esta propuesta que pueden ser utilizados en la práctica. Estos aspectos son: interacción a través de solución de problemas; interacción colectiva; introducción de los esquemas simbólicos generalizados desde el inicio a partir del uso de las fórmulas en todas las ciencias.

Se propone que los niños aprendan a partir del proceso de resolución de problemas que los maestros plantean. La solución de problemas necesariamente requiere de uso de conceptos del área de conocimientos que los maestros trabajan. Los problemas son sistematizados y permiten conducir a la formación de los conceptos en las ciencias. El planteamiento de problemas y su resolución se plantean como una colaboración entre el maestro y todo el grupo de los alumnos. Todos los alumnos juntos buscan la solución del problema. El maestro introduce los signos y las fórmulas, a través de las cuales los problemas se pueden solucionar (NICOLA y TALIZINA, 2017; DAVIDOV, 1996, 2000). Esta solución colectiva de problemas es esencial para la teoría de la actividad, ante la cual la enseñanza implica un análisis sistémico y unitario de un proceso real de solución de problemas que se establecen ante un hombre histórico-social quien es el sujeto de su propia actividad (TALIZINA, 2018; SOLOVIEVA, 2019).

\section{Ventajas de la teoría de la actividad para el proceso de enseñanza- aprendizaje}

La ventaja del enfoque de la teoría de la actividad para la educación se observa desde una clara aportación para el diseño e implementación de los objetivos y los métodos de la enseñanza que garantiza el aprendizaje de los alumnos y, de esta forma, conduce a su desarrollo psicológico. La aplicación de la concepción de Elkonin-Davidov al campo educativo, junto con el estudio general de la teoría de la actividad aplicada a la enseñanza puede ayudar a superar los errores más graves que se parecian actualmente en el sistema educativo en muchos países de América Latina: 
1) incluir a los conceptos teóricos generales de cada materia escolar como unidades de aprendizaje, lo cual implica una re-sistematización completa de las materias escolares;

2) utilizar a las acciones intelectuales en lugar de insistir en el uso constante de acciones prácticas y cotidianas, lo cual empobrece una motivación cognitiva de los alumnos y solo los enfoca en las competencias prácticas;

3) identificar a los conceptos teóricos generales y particulares para valorar los éxitos de aprendizaje y planear el sistema de apoyo para los alumnos con bajo éxito escolar;

4) identificar las acciones intelectuales generales y particulares para la realización de las prácticas y las unidades de trabajo pedagógico para cada materia y cada ciclo escolar.

Desde la propuesta educativa de la teoría de la actividad, que puede ser comprendida con el nombre de la "enseñanza que conduce al desarrollo" es necesario que los maestros comprendan y les transmitan a los alumnos que las características esenciales de los conceptos científicos son el resultado de procesos de abstracción y generalización de las diversas características que tienen los objetos y fenómenos del mundo. Además, la organización especial de la metodología de la enseñanza se debe considerar como un proceso creativo, continuo y flexible y que no puede existir un final de este proceso, tal y como no pude existir un final en la creación de la tecnología y el arte en la humanidad. La experimentación e la innovación en la metodología de la enseñanza debe ser tratada como algo permanente y dinámico. Cualquier falla o deficiencia de la enseñanza en el nivel anterior, no sólo se mantiene a lo largo de todo el proceso educativo, sino que agrava cada vez más, el aprendizaje del alumno.

Desde la teoría de la actividad, que incluye la postura de Elkonin-Davidov y la de Galperin, se puede argumentar un método global para la reorganización del proceso educativo. Proponemos llamar a este método como "método de organización sistémica y orientada" del proceso educativo. Dicho método implica los elementos necesarios y suficientes, lo cual implica una reorganización del proceso educativo. Esta concepción permite: 
1) organización sistémica de cada materia escolar de lo general a lo particular;

2) elección de los conceptos generales con los que se debe iniciar la formación de pensamiento teórico para cada materia escolar y para cada sección de la materia;

3) diseño y planeación de las acciones intelectuales de los alumnos de acuerdo a la teoría de la interiorización;

4) diseño y planeación de la orientación externa particular que les permita a los alumnos realizar correctamente las acciones intelectuales generale y específicas;

5) organización de los tipos de comunicación entre los docentes y alumnos,

6) elección de los temas concretas y las formas de evaluación apropiados para cada nivel educativo.

\section{Consideraciones críticas}

En base a lo expuesto anteriormente, queremos expresar que no tiene mucho sentido considerar a la concepción de la "enseñanza desarrolladora" como una concepción fuera de la teoría de la actividad y sin ninguna relación con la propuesta de Galperin. Para ellos, expresaremos varios argumentos.

En primer lugar, V.V. Davidov fue alumno de doctorado y colaborador activo de P.Ya. Galperin. En sus libros, Davidov constantemente cita a los representantes de la teoría de la actividad de una forma consistente y positiva, entre ellos cita a Galperin en la mayoría de sus publicaciones.

En segundo lugar, aunque no hubiese tales citas, toda la lógica de exposición de Davidov consiste en presentar al proceso de la enseñanza-aprendizaje como una actividad rectora de la edad escolar que tiene su propio contenido y estructura. Las ideas de Davidov (1998) y Talizina $(2018,2019)$ plenamente coinciden en estos textos: es la actividad reflexiva y compartida de enseñanza-aprendizaje dirigido.

Ambos autores afirman que el alumno no inicia la edad escolar con la actividad conformada de enseñanza-aprendizaje, sino que esta se forma gradualmente. Al mismo tiempo, es posible observar ciertas diferencias que 
pueden ser significativas o secundarias, lo cual, desde nuestro punto de vista, dependen de la posición de partida para considerar estas diferencias.

Uno de estos puntos de vista es tomar estas diferencias como acentos o como un interés predominante de los autores. En este sentido es absolutamente válido considerar que los acentos de diversas investigaciones frecuentemente no coinciden sin que sean necesariamente diferentes enfoques (SOLOVIEVA y COLS., 2020). Por ejemplo, desde la teoría de la actividad es posible estudiar la estructura (TALIZINA, 2018) o el contenido de algunas materias de la actividad de enseñanza-aprendizaje (SOLOVIEVA, 2016; SOLOVIEVA y QUINTANAR, 2018b); es posible hacer acento en los motivos de los alumnos (MATA, 2020) o en los motivos de los maestros (MORALES y SOLOVIEVA, 2020).

¿Cuáles son estos acentos?

En la postura de Galperin y, consecuentemente de Talizina, sin duda alguna, el acento se hace en la organización del proceso de interiorización o de la formación de los conceptos dentro de las acciones intelectuales. Estos autores con claridad presentan las etapas formativas, por las cuales se sugiere pasar durante esta formación. Se trata de la etapa de motivación, presentación de la base orientadora de la acción, etapa de la acción materializada, etapa de la acción oral externa y de la acción mental (interna). Toda esta propuesta de ninguna manera excluye la posibilidad de trabajar con la presentación de los problemas creativos e interesantes para los alumnos. Es más, la concepción considera y propone la vía para la formación de la acción de creación de los problemas nuevos (NICOLA y TALIZINA, 2017; ROSAS y SOLOVIEVA, 2017).

En el orden de la presentación de esta concepción los problemas creativos aparecen como un logro avanzado del proceso formativo y no como su elemento inicial. Al mismo tiempo, desde la etapa de motivación se presupone la posibilidad de presentación de un problema atractivo a los alumnos. Al mismo tiempo, el problema atractivo no es el elemento central de la concepción, sino un elemento complementario. El lugar del elemento central de toda la concepción ocupa el elemento de la "base orientadora de la acción". Galperin (2000) escribe que de la orientación depende toda la acción, su solución y su 
verificación. En todos los momentos se plantea la actividad compartida y coordinada entre los alumnos y el maestro. La concepción de Davidov y sus seguidores cambia los acentos en el proceso de la enseñanza. El lugar central ocupa el elemento de situación de problema, el cual se les plantea a los alumnos. No se insiste en los pasos operativos de la formación de la acción intelectual, pero una lectura profunda de los textos de Davidov permite ver que el problema es una acción intelectual que se introduce y que se realiza en el trabajo colectivo de los alumnos. El tema de la base orientadora de la acción no se plantea con insistencia, pero de ninguna forma la descarta.

No descartamos la posibilidad que durante la lectura de los textos de Davidov puede surgir la idea de un proceso libre por parte de los alumnos, pero esta impresión desaparece ante una lectura profunda. Se trata de un proceso colectivo de solución de los problemas. No se insisten en los planos de presentación de estos problemas.

En la concepción de Elkonin-Davidov, el lugar central ocupa el problema creativo que los alumnos solucionan, mientras que el lugar central en el sistema de Galperin ocupa el concepto de la orientación. Esta orientación es necesaria también para la solución y creación de los problemas intelectuales, además, de que la presentación de problemas intelectuales debe seguir un orden formativo. Este orden también sigue la misma idea de presentar primero los conocimientos teóricos generales y más adelante los conocimientos más particulares.

Desde nuestro punto de vista, la concepción de Davidov-Elkonin aporta mucho para la organización del proceso educativo no como una concepción aislada, pero siempre y cuando se considere como un elemento dentro de la teoría de la actividad. Si se nos preguntara, si es importante o no considerar la propuesta de presentación de los problemas atractivos y creativos a los alumnos, debemos contestar: "si". Si se nos preguntara si vale la pena realizar esto a costo de exploración de la base orientadora de la acción y de las etapas de la formación de la acción, debemos contestar: "no". Unicamente la combinación de las aportaciones de ambas concepciones puede llevar al éxito de organización del proceso educativo innovador como una alternativa a la “enseñanza tradicional” y "la escuela activa". 


\section{Implicaciones metodológicas y prácticas}

Los autores de este artículo han tenido la experiencia de desarollar la metodología de la enseñanza desde la teoría de la actividad en México y aplicarla en el nivel preescolar y escolar en una institución educativa particular. Esta experiencia muestra la utilidad de las aportaciones de la teoría de la actividad en general, más allá de las concepciones estrechas cada una de las cuales aporta, sin duda alguna, elementos valiosos para la organización de proceso de la enseñanzaaprendizaje. Consideramos, que en nuestro trabajo estamos utilizando principalmente las aportaciones del sistema de Galperin, lo cual no contradice a la utilización de la aplicación de resolución de los problemas creativos y reflexivos de acuerdo al sistema de Elkonin-Davidov.

En la organización del proceso educativo en la edad escolar se realiza la introducción de las acciones intelectuales generales y específicas (Talizina, 2019).

La mejor manera de ofrecer las acciones intelectuales consiste en la organización de las acciones compartidas colectivas entre todos los participantes del salón de clases y evitar las acciones individuales de cada uno de ellos por separado. El maestro participa como orientador y participante activo clave en todas las acciones de los alumnos. La acción es un concepto teórico fundamental de la teoría de la actividad dentro de area de psicología y tiene que ver con la concepción del desarrollo histórico-cultural del ser humano. La acción se comprende como una unidad y elemento central de la actividad cultural (SOLOVIEVA, 2014). La actividad de aprendizaje compartido y dirigido es uno de los ejemplos de esta actividad cultural intelectual que fue estudiada con detalles por los representantes de la teoría de la actividad (RUBINSTEIN, 1998; GUIPPENREITOR, 1996; TALIZINA, 1984, 2018, 2019). Nosotros consideramos que la solución de problemas intelectuales creativos, que se proponen en el sistema de Elkonin-Davidov, sin duda alguna, se deben considerar como un tipo de las acciones intelectuales.

El elemento central de la acción y de la actividad es su motivo (LEONTIEV, 1993). Es fundamental que los maestros logren identificar el 
motivo cognoscitivo de sus alumnos como el elemento central de sus acciones de aprendizaje escolar. En las publicaciones recientes se ha mostrado que la organización de la enseñanza de acuerdo al sistema educativo de ElkoninDavidov garantiza la motivación interna positiva en los alumnos más elevada en comparación con la motivación de los alumnos que aprenden en las escuelas tradicionales (GORDEEVA, 2018).

Todas las actividades intelectuales, en nuestro sistema de educativo, se realizan de forma grupal, con todos los niños de forma simultánea, por lo que prácticamente no se usan no las individuales en las sesiones escolares. En todas las ocasiones el adulto es un participante activo y orientador motivante para las actividades, las cuales poseen una planeación sistémica que se basa en el proceso de interiorización desde el plano externo, garantizando el paso gradual al plano interno de la acción del niño (QUINTANAR y SOLOVIEVA, 2020).

En todas las materias que se imparten en la escuela primaria se ha realizado una reestructuración total de las materias como sistemas de conocimientos. Dichos sistemas se organizan desde lo general a lo particular. Con lo general se comprenden los conceptos nucleares básicos de cada materia (sistema de conocimientos), junto con las acciones que implican el uso de los mismos conceptos (TALIZINA, 2019). Los conceptos sistémicos nucleares se deben de introducir desde el inicio mismo de las materias escolares dentro de las acciones intelectuales correspondientes. Lo anterior significa que el maestro debe conocer y utilizar la orientación adecuada para la introducción de las acciones con los conceptos nucleares de lingüística, matemáticas, ciencias sociales y naturales (QUINTANAR y SOLOVIEVA, 2020). De esta forma, se trabaja con el pensamiento teóricoreflexivo desde la escuela primaria.

Los conocimientos teóricos iniciales adquiridos por los alumnos les deben ayudar a penetrar en los aspectos más sofisticados de las ciencias en los grados escolares siguientes. En cada momento, el maestro debe elaborar las orientaciones apropiadas para todos los conceptos y mostrar su uso en las acciones intelectuales. Por ejemplo, los conceptos lingüísticos adquiridos se pueden utilizar durante el trabajo con la lectura y análisis de textos literarios narrativos, artísticos y 
descriptivos (científicos). La complejidad estructural de los textos literarios se eleva de un grado de primaria a otro y se complejizan igualmente los elementos literarios que se analizan: contenido, estructura y género literario (SOLOVIEVA, 2016; SOLOVIEVA y QUINTANAR, 2020 b).

\section{Conclusiones}

La concepción de Elkonin-Davidov sobre la enseñanza-aprendizaje que conduce al desarrollo se debe considerar como una concepción particular sobre la actividad rectora de los estudios escolares dentro de la teoría de la actividad. Desde esta concepción se establece el objetivo psicológico del desarrollo de los alumnos: formar en ellos una visión teórica del mundo a través de la enseñanza teórico-reflexiva (DAVIDOV, 2008). Dicho objetivo no figura en los objetivos de la enseñanza tradicional (conductista o constructivista), mientras que precisamente este se plantea como objetivo psicológico central de la edad escolar por parte de los exponentes de la teoría de la actividad (DAVIDOV, 2008; TALIZINA, 2019; TALIZINA, SOLOVIEVA y QUINTANAR, 2017). La teoría de la actividad, de acuerdo a lo expuesto, permite justificar la elaboración y aplicación de métodos innovadores implica una constante formación y aprendizaje propio de los docentes de todos los niveles educativos. La elaboración de los métodos de enseñanza-aprendizaje no debe proceder de forma caótica y empírica y sometida a "modas", sino debe ser argumentada desde una teórica sólida que engloba al proceso de enseñanza-aprendizaje en una visión global del desarrollo cultural del alumno (DAVIDOV, 1996; OBUKHOVA, 2006; FARIÑAS, 2020; TALIZINA, SOLOVIEVA y QUINTANAR, 2010; SOLOVIEVA, 2019). La participación del adulto orientador en este proceso es enorme, lo cual requiere de una previa preparación y participación creativa constante.

La teoría de la actividad permite comprender al proceso educativo como de un proceso cultural creativo y dialéctico que puede y debe ser estudiado, modificado y formado simultáneamente a través de los esfuerzos de sus participantes. 


\section{Referencias}

DAVIDOV, V. V. Tipos de generalización en la enseñanza. Moscú: Sociedad Pedagógica Rusa. 2000.

DAVIDOV, V.V. La teoría de la enseñanza que conduce al desarrollo. Moscú: INTER. 1996.

DAVIDOV, V.V. (2008). Conferencias sobre la psicología general. Moscú: Academia. 2008.

DE MORAES, M. Estudio de la actividad voluntaria en niños de educación infantil de acuerdo la teoría histórico-cultural. Tesis Doctoral. Universidad Federal de Sao Carlos. 2018.

ELKONIN, D.B. Obras psicológicas escogidas. Moscú: Pedagogía. 1989.

FARIÑAS, G. La autonomía como indicador del desarrollo de la personalidad. Obutchénie: Revista De Didática E Psicologia Pedagógica, 4(1), 86106. 2020. DOI: https://doi.org/10.14393/OBv4n1.a2020-56473.

GALPERIN, P. Y. Actividad psicológica como ciencia objetiva. Moscú: Academia de Ciencias Pedagógicas y Sociales. 1998.

GALPERIN, P.Ya. Cuatro conferencias sobre psicología. Moscú, Escuela Superior. 2000.

GUIPPENREITOR, Y. Introducción a la psicología general. Moscú: Universidad Estatal de Moscú. 1996.

GONZÁLEZ-MORENO, C; SOLOVIEVA, Y. Impacto de juego de roles sociales en la formación de la función simbólica en preescolares. Revista de Psicología de la Universidad de Antioquía. 8 (2): 49-70. 2016.

GONZÁLEZ-MORENO, C; SOLOVIEVA, Y. Evaluación de las neoformaciones de la edad preescolar en niños colombianos. Revista de Psicología Universidad de Antioquia, 11(2), 7-44. 2019. DOI: https://doi.org/10.17533/udea.rp.v11n2a01.

GORDEEVA, T; SYCHEVB, O; PSHENICHNUKA, D; SIDNEVAA, A. Academic Motivation of Elementary School Children in Two Educational Approaches - Innovative and Traditional. Psychology in Russia: State of the Art. Volume 11 (4), 22-39. 2018.

ILIENKOV, E.V. Problema de lo ideal: historia y contexto. En: V.I. Tolstih. Eval. Vasilievich Ilienkov. Filosofia de segunda mitad del siglo XX. Moscú: ROCCPEN: 153-240. 2009. 
LEONTIEV, A. N. Actividad, Conciencia y Personalidad. México: Cartago. 1993.

MATA, A. Los motivos del aprendizaje escolar en primaria. Tesis para la obtención del grado de Doctorado. Puebla, México: Universidad Iberoamericana de Puebla. 2020.

MORALES, A; SOLOVIEVA, Yu. Professional motives in primary school teachers. In: J. Glozman \& N. Korepina. Proceedings of Science School: $2^{\text {nd }}$ International Neuropsychological Summer School named after A. R. Luria "The World After the Pandemic: Challenges and Prospects for Neuroscience”. Ministry of Science and Higher Education of the Russian Federation, Ural Federal University. Ekaterinburg: Ural Univ. Press: 77-79. 2020.

NIKOLA, G; TALIZINA, N. La formación de las habilidades generales para la solución de los problemas matemáticos. En: Talizina, N.F., Solovieva, Yu. y Quintanar, L. Enseñanza de las matemáticas desde la teoría de la actividad. México: CEIDE: 129-192. 2017.

OBUKHOVA, L.F. Psicología del desarrollo por edades. Moscú, Educación Superior. 2006.

PUENTES, R. V.; PRUDENTE AMORIM, P; COELHO CARDOSO, C. Developmental didactic of actvity: V.V. Repkin's contributions to the ElkoninDavidov system. Fundamental and applied researches in practice of leading scientific schools, 27(3), 188-195. 2018. DOI:

https://doi.org/10.33531/farplss.2018.3.22.

PUENTES, R. V.; LONGAREZI, A. Sistemas didácticos desenvolvimentais: precisões conceituais, metodológicas e tipológicas. Obutchénie. Revista De Didática E Psicologia Pedagógica, 4(1), 201-242. 2020. DOI: https://doi.org/10.14393/OBv4n1.a2020-57369.

QUINTANAR, L., SOLOVIEVA, YU. Importancia de la teoría de la actividad. En: Covarrubias, V. Bases para la introducción y el desarrollo del pensamiento científico en la niñez y la preadolescencia. 111-172. Puebla: CONCYTEP. 2020.

ROSAS, YO; SOLOVIEVA, Yu. Organización de la enseñanza de la solución de problemas aritméticos: trabajo con maestros de primaria. Obucheniye. Revista de la didáctica y Psicología Pedagógica. V 2 (3): 723-739. 2018. DOI:

https://doi.org/10.14393/OBv2n3.a2018-47442.

ROSAS, YO; SOLOVIEVA, Yu. Trabajo con solución de problemas matemáticos en tercer grado de primaria: análisis de dos escuelas privadas. Ensino em Revista. 26 (2): 415-436. 2019. DOI: https://doi.org/10.14393/ER-v26n2a2019-6.

RUBINSHTEIN, S.L. Bases de psicología general. San Petersburgo, Piter. 1998. 
SIDNEVA, A. Developmental effects of Davidov's mathematics curriculum in relation to school readiness and teacher experience. Frontiers in Psychology. 11 (1): 1-10. 2020. DOI: https://doi.org/10.3389/fpsyg.2020.603673.

SOLOVIEVA, YU; QUINTANAR, L. Evaluación del desarrollo simbólico en niños preescolares mexicanos. Cultura y Educación. 25 (2): 167-182. 2013. DOI: https://doi.org/10.1174/113564013806631273.

SOLOVIEVA, Y. La actividad intelectual en el paradigma histórico-cultural. México: CEIDE. 2014.

SOLOVIEVA, Yu. Estrategias introductorias del lenguaje escrito en el idioma inglés. México: Plaza y Valdés. 2015.

SOLOVIEVA, Yu. Enseñanza del lenguaje escrito. Mexico: Trillas. 2016.

SOLOVIEVA, Y.; QUINTANAR, L. La actividad de juego en la edad preescolar. México: Trillas. 2016.

SOLOVIEVA, Y.; QUINTANAR, L. Teoría de Galperin: Orientación para psicología y neuropsicología. En: I. Beltrán Nuñez y B. Leite Ramalho. Galperin y la teoría de la formación planeada por etapas de las acciones mentales y de los conceptos. Investigaciones y las experiencias para una enseñanza innovadora. Campinas, Mercado de Letras. 2018a.

SOLOVIEVA, Y.; QUINTANAR, L. Enseñanza de la lectura. México: Trillas. 2018b.

SOLOVIEVA, Y; QUINTANAR, L. La metodología formativa en la psicología histórico cultural. Madrid, GIUNTI-EOS. 2019.

SOLOVIEVA, Y. Las aportaciones de la teoría de la actividad para la enseñanza. Educando para educar. 37 (1): 13-25. 2019.

SOLOVIEVA, YU; QUINTANAR, L. Las acciones mentales y el problema de las etapas de su formación: siguiendo a Galperin y Talizina. Obucheniye. Revista de la didáctica y Psicología Pedagógica. 4 (1): 59-85. 2020a. DOI:

https://doi.org/10.14393/obv4n1.a2020-56472.

SOLOVIEVA, YU; QUINTANAR, L. Enseñanza y desarrollo de la comprensión y la producción del lenguaje. México: CONCYTEP. $2020 \mathrm{~b}$.

SOLOVIEVA, YU; GONZÁLES-MORENO, C; ROSAS, YO; MATA, A. y MORALES, A. Resultados de investigación educativa desde el modelo históricocultural y la teoría de la Actividad en la Universidad Iberoamericana de Puebla. Ensino em Re-vista. 27 (n. Especial) 1256-1274. 2020. DOI:

https://doi.org/10.14393/ER-v27nEa2020-4. 
TALIZINA, N. La dirección del proceso de asimilación de conocimientos. Moscú, Universidad Estatal de Moscú. 1984.

TALIZINA, N; SOLOVIEVA, Y; QUINTANAR, L. La aproximación de la actividad en psicología y su relación con el enfoque histórico-cultural de L.S. Vigotsky. Novedades educativas, 22, 230: 4-9. 2010.

TALIZINA, N; SOLOVIEVA, Y; QUINTANAR, L. Enseñanza de las matemáticas desde la teoría de la actividad. México: CEIDE. 2017.

TALIZINA, N. La teoría de la enseñanza-aprendizaje de acuerdo con la concepción de la actividad. Moscú: Universidad Estatal de Moscú. 2018.

TALIZINA, N. La teoría de la actividad aplicada a la enseñanza. Benemérita Universidad Autónoma de Puebla. México: BUAP. 2019.

VORONTSOV, A. Compilación de programas aproximados para la escuela primaria. Moscú, BINOM. 2019.

VIGOTSKY, L.S. Obras escogidas. Tomo I. Madrid: Visor. 1991.

VIGOTSKY, L.S. Obras escogidas. Tomo II. Madrid: Visor. 1992.

VIGOTSKY, L.S. Obras escogidas. Tomo III. Madrid: Visor. 1995.

VIGOTSKY, L.S. Problemas de la psicología infantil, en Obras escogidas. Tomo IV. Madrid: Visor. 1996. 